\title{
Optimal spot market inventory strategies in the presence of cost and price risk
}

\author{
X. Guo • P. Kaminsky • P. Tomecek • M. Yuen
}

Received: 11 January 2010 / Accepted: 26 October 2010 / Published online: 19 November 2010 C) The Author(s) 2010. This article is published with open access at Springerlink.com

\begin{abstract}
We consider a firm facing random demand at the end of a single period of random length. At any time during the period, the firm can either increase or decrease inventory by buying or selling on a spot market where price fluctuates randomly over time. The firm's goal is to maximize expected discounted profit over the period, where profit consists of the revenue from selling goods to meet demand, on the spot market, or in salvage, minus the cost of buying goods, and transaction, penalty, and holding costs. We first show that this optimization problem is equivalent to a two-dimensional singular control problem. We then use a recently developed control-theoretic approach to show that the optimal policy is completely characterized by a simple price-dependent two-threshold policy. In a series of computational experiments, we explore the value of actively managing inventory during the period rather than making a purchase decision at the start of the period, and then passively waiting for demand. In these experiments, we observe that as price volatility increases, the value of actively managing inventory increases until some limit is reached.
\end{abstract}

\footnotetext{
X. Guo $\cdot$ P. Kaminsky $(\varangle) \cdot$ M. Yuen

Department of Industrial Engineering and Operations Research, University of California,

Berkeley, CA 94720-1777, USA

e-mail: kaminsky@ieor.berkeley.edu

X. Guo

e-mail: xinguo@ieor.berkeley.edu

M. Yuen

e-mail: ming@ieor.berkeley.edu

P. Tomecek

J.P. Morgan, New York, NY, USA

e-mail: pascal.i.tomecek@jpmorgan.com
} 
Keywords Inventory $\cdot$ Continuous time $\cdot$ Spot market $\cdot$ Singular control $\cdot$ Pricing

\section{Introduction}

Spot market supply purchases are increasingly considered an important operational tool for firms facing the risk of higher than anticipated demand for goods (see, e.g., Simchi-Levi et al. 2008 and the references therein). For example, Hewlett-Packard manages the risks associated with electronic component procurement by utilizing a portfolio of long term and option contracts and the spot market (Billington 2002). Indeed, there has been a recent stream of research focusing on determining an optimal mix of long term fixed commitment and options/procurement contracts. In these models, the spot market is typically employed if supply requirements exceed the contracted amount of the fixed commitment contract, or if the spot price happens to be lower than the exercise price of the procurement options.

In this paper, we demonstrate that if effectively utilized, the spot market can be used to hedge against much more than just excess demand. In many cases, the spot market can be a powerful tool for hedging against both supply cost uncertainty and demand price uncertainty in the supply chain, even without an accompanying portfolio of supply contracts. To explore this concept, we develop a stylized model of a firm that has a random period of time to increase or decrease inventory by purchasing or selling on the spot market before facing a single demand of random magnitude, the revenue of which is a function of the spot market price when the demand is realized. Utilizing a novel control-theoretic approach and employing recently developed solution techniques, we demonstrate that in many cases, the firm can use purchases and sales on the supply spot market to increase expected profits, and thus to guard against both low prices for its products and high prices for its product components.

As an illustration, consider a firm that owns electric power storage technology. Initially, firms considered such technology as a way to improve the efficiency of electricity generation technology, but as observed in Schainker (2004), "utilities are beginning to look at the advantages of operating a storage plant more strategically .... Some utilities are now viewing storage plants in an opportunistic manner .... [O]ne benefit is a matter of buying low and selling high .... An energy storage plant puts a utility in a position to buy electricity when it is cheapest ...." Thus, consider a utility that purchases electricity on the spot market in anticipation of future demand. At any time, the utility can increase (or decrease) its inventory of power utilizing the spot markethowever, at some point, it needs to provide this electricity to its customers. Such a firm needs an effective policy for managing its inventory of electric power in anticipation of future demand. This is the problem we consider in this paper.

\section{Literature review and our work}

There is a long history of research focusing on inventory strategies when the cost of the inventory is random, typically with the objective of minimizing inventory cost. Various researchers (Kalymon 1971; Sethi and Cheng 1997; Cheng and Sethi 1999; Chen and Song 2001, for example) considered versions of periodic review models where 
component costs (and sometimes other problem parameters) are Markov-modulated, usually demonstrating the optimality of state dependent basestock or $(\mathrm{s}, \mathrm{S})$ policies. Another stream of literature modeled deterministic demand and characterized optimal policies, either in a periodic model with random raw material prices (Golabi 1985; Berling 2008), increasing (Gavirneni and Morton 1999), or decreasing (Wang 2001) prices, or with one or two different levels of constant continuous time demand and occasional supply price discounts (Moinzadeh 1997; Goh and Sharafali 2002; Chaouc 2006).

Finally, a growing stream of research considers the impact of a spot market on supply chain operations. Haksoz and Seshadri (2007) survey this work, the bulk of which model the spot market with a single spot market price or a discretely realized series of spot market prices, and typically allow one opportunity to buy or sell on the spot market following each demand realization. One exception is Li and Kouvelis (1999), who modeled a situation in which deterministic demand must be met after a deterministic time period, but the firm has a contract to procure supply on the spot market at some point before demand is realized, where spot market price is a continuous random process. In that paper, the optimal purchase time was derived numerically.

Our work: We consider a continuous time model of spot market price evolution, and determine how the firm can buy and sell in the spot market repeatedly in order to guard against both supply cost uncertainty and demand price uncertainty. Specifically, we model the inventory level of the firm at time $t, Y_{t}$, with a pair of controls $\left(\xi_{t}^{+}, \xi_{t}^{-}\right)$so that $Y_{t}=Y_{0}+\xi_{t}^{+}-\xi_{t}^{-}$. Here $\xi_{t}^{+}$and $\xi_{t}^{-}$are non-decreasing processes and represent respectively the total accumulated inventory ordered and sold by time $t$ starting from time 0 . We assume that the price of each unit of inventory is stochastic and modeled by a Brownian motion process as in Haksoz and Seshadri (2007) and Li and Kouvelis (1999). We also assume that the time until the demand arrives, as well as the amount of that demand, is random. The revenue associated with the demand is assumed to be a function of the amount of that demand and the spot market price at the time when the demand arrives. In addition to the running holding cost, there are costs whenever inventory level is increased or decreased by selling or buying at the spot market: this adjustment cost is a function of the spot price and the amount of the adjustment, plus a proportional transaction cost. Note that this cost can be negative when selling inventory. Given this cost structure, the goal is to maximize expected discounted profit over an infinite time horizon. To facilitate our analysis, we assume no fixed cost and focus on explicitly characterizing the optimal policy.

In particular, we show that the optimal inventory policy depends on both the spot price and inventory level, and that it is in principle a simple and not necessarily continuous $(F, G)$ policy. Given a spot price $p$ and inventory level $z$, if $(p, z)$ falls between $(F(z), G(z))$, no action is taken; if $(p, z)$ falls above $F(z)$ (below $G(z)$ ), the inventory level is reduced to $F(z)$ (raised to $G(z)$ ).

Our model is closely related to a stream of research (Bather 1966; Archibald 1981; Constantinides and Richard 1978; Harrison 1983; Harrison and Taksar 1983; Taksar 1985; Sulem 1986; Ormeci et al. 2006) focusing on continuous time inventory models via impulse controls (i.e. with a fixed cost) or singular controls (i.e. without a fixed cost) formulation. Most of these papers (with the exception of Archibald (1981) where 
the demand process is Poisson) considered a one product inventory model where the inventory level is a controlled Brownian motion. That is, the inventory level without intervention was modeled by a Brownian motion, and the continuous adjustment of the inventory level was assumed additive to the Brownian motion and with a linear cost plus a possibly fixed cost. Subject to an additional holding cost and shortage penalty, the objective in these papers was to minimize either the expected discounted cost or the average cost (Bather 1966; Ormeci et al. 2006) over an infinite time horizon. Except for Taksar (1985) and Ormeci et al. (2006), most of the models assumed no constraints on the inventory level besides restricting it to the positive real line. Assuming a fixed cost, Constantinides and Richard (1978) proved the existence of an optimal $(d, D, U, u)$ policy for this system: do nothing when inventory is in the region of $(d, u)$, and adjust the inventory level to $D$ (or $U$ ) whenever the inventory level falls to $d$ (or rises to $u$ ). This optimal policy and the solution structure were more explicitly characterized under various scenarios in Harrison and Taksar (1983), Harrison (1983), Taksar (1985), Sulem (1986) and Ormeci et al. (2006).

The main contribution of our paper is best discussed in light of several crucial elements underlying all previous control-theoretic inventory analysis. First, the price of the inventory was typically assumed to be constant so that the cost of the inventory control was linear. Secondly, the inventory control was usually additive to a Brownian motion, and as a result the inventory level was either unconstrained on the positive real line, or an infinite penalty cost was needed to ensure an upper bound on the inventory level (Taksar 1985; Ormeci et al. 2006). These two characteristics ensured the control problem to be one-dimensional, to facilitate the analysis of the value function. The solution approach was to apply the Dynamic Programming Principle and to solve some form of Hamilton-Jacobi-Bellman equations or Quasi-Variational-Inequalities, with a priori assumptions on the regularity conditions.

In contrast, in our model, the adjustment cost is no longer linear in the amount of adjustments, but instead depends on the spot price, the transaction cost, and the amount of adjustment, and the inventory control variable is modeled directly, and is no longer necessarily additive to the underlying Brownian motion process. Thus, lower and upper bounds on the inventory level (that is, capacity constraints) are modeled directly. This approach to modeling capacity constraints has an additional advantage it can be easily extended to more complex constraints on inventory levels without further technical difficulty. In essence, the introduction of price dynamics leads to a higher dimensional singular control problem for which previous analysis cannot be directly generalized. The derivation in this paper is thus based on a new solution approach, which allows us to bypass the possible non-regularity of the value functions. The key idea is to break down the two-dimensional control problem by "slicing" it into pieces of one-dimensional problem, which is an explicitly solvable two-state switching problem, and to show that this re-parametrization is valid by the notion of "consistency" established in Guo and Tomecek (2008).

This paper is most closely related to Guo and Tomecek (2008) and Merhi and Zervos (2007). Indeed, we utilize techniques first developed in Guo and Tomecek (2008), although we believe that the use of these techniques is by no means straightforward or immediately obvious. More importantly, via the theory of stochastic differential equations, we are able to provide an important insight into the inventory model we are 
analyzing and potentially a broader class of related models: it turns out that our model, a very natural generalization of the standard newsvendor model, is in fact a mathematically sophisticated continuous time two-dimensional stochastic control problem. This provides a direct mathematical explanation as to why inventory models integrating stochastic prices and the option of utilizing a spot market are fundamentally difficult. Also, although our continuous time model is close to that of Merhi and Zervos (2007), the first paper in our knowledge to explicitly solve a similar two-dimensional control problem, their solution approach is the traditional guess-and-proof verification theorem approach and requires a priori regularity conditions for the value function. In contrast, our approach requires minimal regularity condition in both the payoff function and the value function, and so is more general. For more discussion of our approach, see Sect. 4.6.

In the next section, we formally introduce our model. In Sect. 4, we translate the problem into an equivalent singular control problem and develop explicit analytical expressions for the optimal policy for this model. In Sect. 5, we computationally explore some of the implications of our results.

\section{The model}

\subsection{The model setting}

We consider a firm that purchases supply from a spot market in which the price of the supply component fluctuates over time. At a random time $\tau$, the firm faces a random customer demand $D$. The firm meets demand if possible (we assume one supply component meets one unit of demand), charging an exogenously determined price that is a function of the spot market component price, and then salvages any excess inventory. At any time $t \in[0, \tau)$, the firm can instantaneously increase inventory of the component up to some upper bound on capacity $b$ or instantaneously decrease inventory down to some lower bound on inventory $a$. However, the firm cannot buy inventory of the component to satisfy demand at time $\tau$, and the firm can only buy inventory a finite number of times in a finite interval. Net gain at time $\tau$ is from selling to arriving customers and liquidating excess inventory minus penalty associated with not meeting demand, and thus is a function of the selling price and the inventory level at time $\tau$, and the demand distribution. Moreover, at any time $t \in[0, \tau)$, inventory increase is associated with the purchase price of per unit at the supply spot market price $P_{t}$, plus possibly additional proportional transaction cost $K^{+}$. Similarly, inventory reduction is associated with the spot market price $P_{t}$, minus possibly additional proportional transaction cost $K^{-}$. Finally, there is a running holding cost for each unit of inventory $C_{h}$.

To capture this scenario in mathematical terms, we start with a complete and filtered probability space $(\Omega, \mathcal{F}, \mathbb{P})$, and assume that the arrival time of the request, $\tau$, is exponentially distribution with rate $\lambda$ (so that the average arrival time is $1 / \lambda$ ). $D$, the random variable representing the demand at time $\tau$ is described by distribution function $F_{D}$. Meanwhile, the component spot market price $\left(P_{t}\right)_{t \geq 0}$ is stochastic and 
its dynamics are governed by a geometric Brownian motion such that ${ }^{1}$

$$
d P_{t}=P_{t}\left(\mu d t+\sqrt{2} \sigma d W_{t}\right), \quad P_{0}=p
$$

Here $W_{t}$ is the standard Brownian motion on the probability space $(\Omega, \mathcal{F}, \mathbb{P})$, and $\mu$ and $\sigma$ represent respectively the expected spot market price appreciation and the potential price risk. We express the net gain at request time $\tau$ by $H\left(Y_{\tau}, D\right) P_{\tau}$, where $Y_{\tau}$ is the inventory level at time $\tau$, and $H\left(Y_{\tau}, D\right)$ represents the revenue multiplier associated with selling each unit of the inventory, as well as a penalty associated with each unit of unmet demand and salvage associated with each unit of excess inventory. Specifically,

$$
H(x, D)=\alpha \min (D, x)+\alpha_{o} \max (x-D, 0)-\alpha_{u} \max (D-x, 0),
$$

where $\alpha \geq 1$ is the mark-up multiplier for each unit of met demand, $\alpha_{u} \geq 0$ is the penalty price multiplier for each unit the firm is short, and $0 \leq \alpha_{o} \leq 1$ is the fraction of price the firm is able to get by salvaging excess inventory.

To define admissible inventory policies, we specify the filtration $\mathbb{F}$ representing the information on which inventory decisions are based. Given $\lambda$ and the distribution of $D$, it is clear that $\mathbb{F}=\left(\mathcal{F}_{t}\right)_{t \geq 0}$ is the filtration generated by $P_{t}$. Given $\mathbb{F}$, we define a pair of left-continuous with right limit, adapted, and non-decreasing processes $\xi_{t}^{+}$ and $\xi_{t}^{-}$to be the cumulative increases and decreases in supply inventory (purchases and sales, respectively) up to time $t$, with $\xi_{0}^{ \pm}=0$. Therefore, $Y_{t}$, the inventory level at time $t \in[0, \tau)$, is given by

$$
Y_{t}=y+\xi_{t}^{+}-\xi_{t}^{-}
$$

where $y$ is the initial inventory amount.

$Y_{t}$ is assumed to be a finite variation process, which implies that the total amount of inventory bought and sold is bounded. Meanwhile, for uniqueness of expression $\left(3,\left(\xi^{+}, \xi^{-}\right)\right.$are supported on disjoint sets. Furthermore, $\xi^{+}$and $\xi^{-}$are adapted to $\mathbb{F}$ implying that the firm is not clairvoyant. $Y$ is left-continuous, capturing the restriction that the commodity cannot be purchased at time $\tau$ to satisfy demand. Also, note that given the upper and lower bounds on capacity discussed above, there exists $0 \leq a<b \leq \infty$ such that an admissible control policy must satisfy $Y_{t} \in[a, b]$ for all $t \leq \tau$. Finally, for well-posedness of the problem, we assume $\mathbb{E}\left[\int_{0}^{\infty} e^{-(r+\lambda) t} d \xi_{t}^{+}+\int_{0}^{\infty} e^{-\left(r_{\lambda}\right) t} d \xi_{t}^{-}\right]<\infty$.

To account for the time value between $[0, \tau]$, we define $r \geq 0$ to be a discount rate. Thus, at time $t \in[0, \tau)$, increases in the inventory incur a cost $-e^{-r t}\left(P_{t}+K^{+}\right) d \xi_{t}^{+}$ per unit, and decreases in the inventory generate revenue $e^{-r t}\left(P_{t}-K^{-}\right) d \xi_{t}^{-}$per unit. In addition, assuming a running holding $\cos t C_{h}$ for each unit of inventory, the holding cost between $(t, t+d t) \subset[0, \tau)$ is $e^{-r t} C_{h} Y_{t} d t$.

\footnotetext{
${ }_{1}^{1}$ The extra term $\sqrt{2}$ is for notational convenience in the main text.
} 
Given this setting and any admissible control policy $\left(\xi^{+}, \xi^{-}\right)$, the expected return to the firm is:

$J\left(p, y ; \xi^{+}, \xi^{-}\right)=$payoff at transaction time $\tau$-running holding cost between [0, $\left.\tau\right]$ - cost of inventory control (via buying and selling) between $[0, \tau]$

$$
\begin{aligned}
= & \mathbb{E}\left[e^{-r \tau} H\left(Y_{\tau}, D\right) P_{\tau}-\int_{0}^{\tau} e^{-r t} C_{h} Y_{t} d t\right. \\
& \left.-\int_{0}^{\tau} e^{-r t}\left(P_{t}+K^{+}\right) d \xi_{t}^{+}+\int_{0}^{\tau} e^{-r t}\left(P_{t}-K^{-}\right) d \xi_{t}^{-}\right] .
\end{aligned}
$$

Assuming that $\tau$ is independent of $\mathbb{F}$ and $D$ is independent of both $\tau$ and $\mathbb{F}$, a simple and standard conditioning argument gives an equivalent form of this expected return:

$$
\begin{aligned}
J\left(p, y ; \xi^{+}, \xi^{-}\right)= & \mathbb{E}\left[e^{-r \tau} H\left(Y_{\tau}, D\right) P_{\tau}-\int_{0}^{\tau} e^{-r t} C_{h} Y_{t} d t\right. \\
& \left.-\int_{0}^{\tau} e^{-r t}\left(P_{t}+K^{+}\right) d \xi_{t}^{+}+\int_{0}^{\tau} e^{-r t}\left(P_{t}-K^{-}\right) d \xi_{t}^{-}\right] \\
= & \mathbb{E} \int_{0}^{\infty} \lambda e^{-(r+\lambda) t} H\left(Y_{t}, D\right) P_{t} d t-\int_{0}^{\infty} e^{-(r+\lambda) t} C_{h} Y_{t} d t \\
& \left.-\int_{0}^{\infty} e^{-(r+\lambda) t}\left(P_{t}+K^{+}\right) d \xi_{t}^{+}+\int_{0}^{\infty} e^{-(r+\lambda) t}\left(P_{t}-K^{-}\right) d \xi_{t}^{-}\right] .
\end{aligned}
$$

\subsection{The optimization problem}

The firm's goal is to manage inventory in order to maximize the expected discounted value over all possible admissible control policies $\left(\xi^{+}, \xi^{-}\right)$. In other words, the firm must solve the following optimization problem:

$$
W(p, y)=\sup _{\left(\xi^{+}, \xi^{-}\right) \in \mathcal{A}_{y}^{\prime}} J\left(p, y ; \xi^{+}, \xi^{-}\right)
$$

subject to

$$
\begin{gathered}
Y_{t}:=y+\xi_{t}^{+}-\xi_{t}^{-} \in[a, b], \quad y \in[a, b], \\
d P_{t}:=\mu P_{t} d t+\sqrt{2} \sigma P_{t} d W_{t}, \quad P_{0}:=p>0, \\
C_{h} \in \mathbb{R}, \quad K^{+}+K^{-}>0 ;
\end{gathered}
$$


$\mathcal{A}_{y}^{\prime}:=\left\{\left(\xi^{+}, \xi^{-}\right): \xi^{ \pm}\right.$are left continuous, non-decreasing processes,

$$
\begin{aligned}
& y+\xi_{t}^{+}-\xi_{t}^{-} \in[a, b], \quad \xi_{0}^{ \pm}=0 ; \\
& \mathbb{E}\left[\int_{0}^{\infty} e^{-\rho t} d \xi_{t}^{+}+\int_{0}^{\infty} e^{-\rho t} d \xi_{t}^{-}\right]<\infty ; \\
& \mathbb{E}\left[\int_{0}^{\infty} e^{-\rho t} P_{t} d \xi_{t}^{+}+\int_{0}^{\infty} e^{-\rho t} P_{t} d \xi_{t}^{-}\right]<\infty .
\end{aligned}
$$

A few remarks about this formulation: First, we assume throughout the paper that $r+\lambda>\mu$ to ensure the finiteness of the value function. Secondly, to avoid an arbitrage opportunity in the market, we assume that $K^{+}+K^{-}>0$. In addition, we assume without loss of generality that $K^{+}>0$, and consider only a bounded inventory level. Finally, for ease of exposition, we fix without loss of generality $a=0$.

Summarizing, throughout the paper we impose the following standing assumptions:

Assumption $1 \rho:=r+\lambda>\mu$.

Assumption $2 K^{+}>0$.

Assumption $3 a=0$ and $[0, b]$ is bounded.

To simplify subsequent notation, we define $m<0<1<n$ to be the roots of $\sigma^{2} x^{2}+\left(\mu-\sigma^{2}\right) x-\rho=0$, so that

$$
m, n=\frac{-\left(\mu-\sigma^{2}\right) \pm \sqrt{\left(\mu-\sigma^{2}\right)^{2}+4 \sigma^{2} \rho}}{2 \sigma^{2}} .
$$

Finally, from the identity $\rho=-\sigma^{2} m n$, we define a useful quantity $\eta$ such that

$$
\eta:=\frac{1}{\rho-\mu}=\frac{-m n}{(n-1)(1-m) \rho}=\frac{1}{\sigma^{2}(n-1)(1-m)}>0 .
$$

\section{Solutions}

\subsection{An equivalent problem $V(p, y)$}

Assuming that $\tau$ is independent of $\mathbb{F}$ and that $D$ is independent of both $\tau$ and $\mathbb{F}$, the one period optimization problem in the previous section can be proved to be equivalent to the following singular control problem over an infinite time horizon by applying theory of stochastic differential equations (SDEs). We refer the readers to Protter (2004) for necessary background for the theory of SDEs. 
Theorem 4 Assume that $\tau$ is independent of $\left(W_{t}\right)_{t \geq 0}$, and $D$ is independent of both $\tau$ and $\left(W_{t}\right)_{t \geq 0}$. The value function $W(\cdot, \cdot)$ in Eq. (5) satisfies

$$
W(p, y)=\left(-\frac{C_{h}}{\rho}+p\right) y+V(p, y)
$$

where $V(\cdot, \cdot)$ is the value function for the following optimization problem

$$
\begin{aligned}
V(p, y)= & \sup _{\left(\xi^{+}, \xi^{-}\right) \in \mathcal{A}_{y}} \mathbb{E}\left[\int_{0}^{\infty} e^{-\rho t} \tilde{H}\left(Y_{t}\right) P_{t} d t-\left(K^{+}+\frac{C_{h}}{\rho}\right) \int_{0}^{\infty} e^{-\rho t} d \xi_{t}^{+}\right. \\
& \left.-\left(K^{-}-\frac{C_{h}}{\rho}\right) \int_{0}^{\infty} e^{-\rho t} d \xi_{t}^{-}\right],
\end{aligned}
$$

with

$$
\begin{aligned}
\tilde{H}(y)= & \lambda \mathbb{E}[H(y, D)]-(\rho-\mu) y \\
= & \lambda\left(\alpha+\alpha_{u}-\alpha_{o}\right)\left[y\left(1-F_{D}(y)\right)-\int_{y}^{\infty} z f_{D}(z) d z\right] \\
& +\lambda\left(\alpha-\alpha_{o}\right) \mathbb{E}[D]+\left(\lambda \alpha_{0}+\mu-r-\lambda\right) y ; \\
Y_{t}= & y+\xi_{t}^{+}-\xi_{t}^{-} \in[0, b], y \in[0, b] ; \\
d P_{t}= & \mu P_{t} d t+\sqrt{2} \sigma P_{t} d W_{t}, \quad P_{0}=p>0 ; \\
C_{h} \in \mathbb{R}, \quad & K^{+}+K^{-}>0 ; \\
\mathcal{A}_{y}= & \left\{\begin{array}{c}
\left(\xi^{+}, \xi^{-}\right): \xi^{ \pm} \text {are left continuous, non-decreasing processes, } \\
y+\xi_{t}^{+}-\xi_{t}^{-} \in[0, b], \xi_{0}^{ \pm}=0 ; \\
\mathbb{E}\left[\begin{array}{c}
\infty \\
\int_{0}^{\infty} e^{-\rho t} d \xi_{t}^{+}+\int_{0}^{-\rho t} d \xi_{t}^{-}
\end{array}\right]<\infty .
\end{array}\right\}
\end{aligned}
$$

Proof First, given any $\left(\xi^{+}, \xi^{-}\right) \in \mathcal{A}_{y}^{\prime}$, from the integration by parts formula,

$$
\mathbb{E}\left[\int_{0}^{\infty} e^{-\rho t} C_{h} Y_{t} d t\right]=\frac{C_{h}}{\rho} y+\frac{C_{h}}{\rho} \mathbb{E}\left[\int_{0}^{\infty} e^{-\rho t} d \xi_{t}^{+}-\int_{0}^{\infty} e^{-\rho t} d \xi_{t}^{-}\right] .
$$

Next, let $Z_{t}=e^{-\rho t} P_{t}$. by Ito's formula, we have

$$
d Z_{t}=-(\rho-\mu) Z_{t} d t+\sqrt{2} \sigma Z_{t} d W_{t}, \quad Z_{0}=p>0 .
$$


Note that $\rho-\mu>0$ implies $\mathbb{E}\left[\int_{0}^{\infty} Z_{s} d s\right]<\infty$. Moreover $\lim _{t \rightarrow 0} Z_{t}=0$ almost surely and in $L^{1}$. Now, from the integration by parts formula in Protter (2004, p. 68) and noting that $Z$ and $Y$ are left continuous,

$$
\begin{aligned}
\int_{0}^{t} Z_{s-} d Y_{s} & =Z_{t} Y_{t}-\int_{0}^{t} Y_{s-} d Z_{s}-[Z, Y]_{t} \\
& =Z_{t} Y_{t}+(\rho-\mu) \int_{0}^{t} Y_{s} Z_{s} d s-\sqrt{2} \sigma \int_{0}^{t} Y_{s} Z_{s} d W_{s}-p y,
\end{aligned}
$$

because the quadratic covariation of $Z$ and $Y$ satisfies $[Z, Y]_{t}=Z_{0} Y_{0}=$ py for all $t$ from the finite variation of $Y$.

In addition, from Protter $\left(2004\right.$, p. 63), the process $\int_{0}^{t} Y_{s} Z_{S} d W_{s}$ is a martingale, so for all $t>0$,

$$
\mathbb{E}\left[\int_{0}^{t} Z_{s} d Y_{s}\right]=\mathbb{E}\left[Z_{t} Y_{t}+(\rho-\mu) \int_{0}^{t} Y_{s} Z_{s} d s\right]-p y .
$$

And since $Z_{t} \rightarrow 0$ in $L^{1}$ and $Y_{t} \in[0, b]$ is bounded, $\lim _{t \rightarrow \infty} \mathbb{E}\left[Z_{t} Y_{t}\right]=0$.

Furthermore, since $\left(\xi^{+}, \xi^{-}\right) \in \mathcal{A}_{y}^{\prime}$ and $Z_{t}$ is integrable, the dominated convergence theorem gives

$$
\begin{aligned}
\mathbb{E}\left[\int_{0}^{\infty} Z_{s} d Y_{s}\right] & =\lim _{t \rightarrow \infty} \mathbb{E}\left[\int_{0}^{t} e^{-\rho s} P_{s} d Y_{s}\right] \\
& =\lim _{t \rightarrow \infty} \mathbb{E}\left[Z_{t} Y_{t}+(\rho-\mu) \int_{0}^{t} Y_{s} Z_{s} d s\right]-p y \\
& =\mathbb{E}\left[(\rho-\mu) \int_{0}^{\infty} Y_{s} Z_{s} d s\right]-p y .
\end{aligned}
$$

Hence,

$$
\mathbb{E}\left[-\int_{0}^{\infty} e^{-\rho t} P_{t} d \xi_{t}^{+}+\int_{0}^{\infty} e^{-\rho t} P_{t} d \xi_{t}^{-}\right]=p y-\mathbb{E}\left[(\rho-\mu) \int_{0}^{\infty} e^{-\rho t} Y_{t} P_{t} d t\right] .
$$

Now, putting all terms together, an easy application of the dominated convergence theorem reveals the statement.

This equivalence statement suggests that the incorporation of the dynamics of the price process leads to a two-dimension singular control problem formulation with 
a state space $(p, y)$, and generalizes the one-dimensional singular control problem studied extensively in Harrison and Taksar (1983).

We reiterate that Merhi and Zervos (2007) is the first paper to deal with a similar two-dimensional singular control problems with explicit solutions, assuming a certain degree of smoothness of the value functions. In this paper, we explicitly solve a more general form of the control problem with no a priori regularity assumptions on the value function.

\subsection{Preliminary analysis}

Proposition 5 (Finiteness of Value Function) $V(p, y) \leq \eta M p+\frac{C_{h}}{\rho} b$, where $M=$ $\sup _{y \in[0, b]}|\tilde{H}(y)|<\infty$, and $\eta$ is as defined in (9).

Proof Let $p>0$ and $y \in[0, b]$ be given. Since $\rho>\mu$ we have

$$
\mathbb{E}\left[\int_{0}^{\infty} e^{-\rho t}\left[\tilde{H}\left(Y_{t}\right) P_{t}\right] d t\right] \leq \mathbb{E}\left[\int_{0}^{\infty} e^{-\rho t}\left[M P_{t}\right] d t\right] \leq \eta M p .
$$

Note that for any given $\left(\xi^{+}, \xi^{-}\right) \in \mathcal{A}_{y},-y \leq \xi_{t}^{+}-\xi_{t}^{-} \leq b-y$. From integration by parts, for any $T>0$,

$$
-\int_{[0, T)} e^{-\rho t} d \xi_{t}^{+} \leq-\int_{[0, T)} e^{-\rho t} d \xi_{t}^{-}+y
$$

which, together with $K^{+}+K^{-}>0$ and $K^{+}>0$, implies

$$
\begin{aligned}
& \mathbb{E}\left[-\left(K^{+}+\frac{C_{h}}{\rho}\right) \int_{0}^{\infty} e^{-\rho t} d \xi_{t}^{+}-\left(K^{-}-\frac{C_{h}}{\rho}\right) \int_{0}^{\infty} e^{-\rho t} d \xi_{t}^{-}\right] \\
& \leq \frac{C_{h}}{\rho} y-\left(K^{+}+K^{-}\right) \mathbb{E}\left[\int_{0}^{\infty} e^{-\rho t} d \xi_{t}^{-}\right] \leq \frac{C_{h}}{\rho} b .
\end{aligned}
$$

Since these bounds are independent of the control, we have

$$
V(p, y) \leq \eta M p+\frac{C_{h}}{\rho} b<\infty
$$

Lemma $6 \tilde{H}(y)$ is concave in y for ANY distribution of $F_{D}$ with finite expectation. In particular,

$$
\tilde{H}\left(y_{2}\right)-\tilde{H}\left(y_{1}\right)=\int_{y_{1}}^{y_{2}} \tilde{h}(z) d z
$$


with $\tilde{h}(y)$ decreasing in $y$, and

$$
\tilde{h}(y):=\lambda\left[\left(\alpha+\alpha_{u}-\alpha_{o}\right)\left[1-F_{D}(y)\right]+\lambda \alpha_{o}+\mu-\rho .\right.
$$

Furthermore,

$$
\mathbb{E}\left[\int_{0}^{\infty}\left|e^{-\rho t} \tilde{H}\left(Y_{t}\right) P_{t}\right| d t\right]<\infty, \quad \mathbb{E}\left[\int_{0}^{\infty}\left|e^{-\rho t} \tilde{h}\left(Y_{t}\right)\right| d t\right]<\infty .
$$

It is worth mentioning that intuitively, function $\tilde{H}(\cdot)$ captures the ultimate potential benefit of carrying inventory over time and its derivative $\tilde{h}(y)$ represents the impact on business of increasing or decreasing inventory levels. We shall see that this $\tilde{h}(\cdot)$ is a key quantity for characterizing optimal policies.

\subsection{Review of key concepts}

Our solution approach relies on a critical lemma connecting the value function of the singular control problem to that of a switching control problem Guo and Tomecek (2008, Theorem 3.7).

Before stating the lemma, we review some of the key concepts used in this paper.

Definition 7 A switching control $\alpha=\left(\tau_{n}, \kappa_{n}\right)_{n \geq 0}$ consists of an increasing sequence of stopping times $\left(\tau_{n}\right)_{n \geq 0}$ and a sequence of new regime values $\left(\kappa_{n}\right)_{n \geq 0}$ that are assumed immediately after each stopping time.

Definition 8 A two-state switching control $\alpha=\left(\tau_{n}, \kappa_{n}\right)_{n \geq 0}$ is called admissible if the following hold almost surely: $\tau_{0}=0, \tau_{n+1}>\tau_{n}$ for $n \geq 1, \tau_{n} \rightarrow \infty$, and for all $n \geq 0, \kappa_{n} \in\{0,1\}$ is $\mathcal{F}_{\tau_{n}}$ measurable, with $\kappa_{n}=\kappa_{0}$ for even $n$ and $\kappa_{n}=1-\kappa_{0}$ for odd $n$.

Proposition 9 There is a one-to-one correspondence between admissible switching controls and the regime indicator function $I_{t}(\omega)$, which is an $\mathbb{F}$-adapted càglàd process of finite variation, so that $I_{t}(\omega): \Omega \times[0, \infty) \rightarrow\{0,1\}$, with

$$
I_{t}:=\sum_{n=0}^{\infty} \kappa_{n} 1_{\left\{\tau_{n}<t \leq \tau_{n+1}\right\}}, \quad I_{0}=\kappa_{0}
$$

Definition 10 Let $\mathcal{I}=(0, b), y \in \overline{\mathcal{I}}$ be given, and for each $z \in \mathcal{I}$, let $\alpha(z)=$ $\left(\tau_{n}(z), \kappa_{n}(z)\right)_{n \geq 0}$ be a switching control. The collection $(\alpha(z))_{z \in \mathcal{I}}$ is consistent if

$$
\begin{aligned}
& \alpha(z) \text { is admissible for Lebesgue-almost every } z \in \mathcal{I}, \\
& I_{0}(z):=\kappa_{0}(z)=1_{\{z \leq y\}}, \text { for Lebesgue-almost every } z \in \mathcal{I},
\end{aligned}
$$


and for all $t<\infty$,

$$
\begin{aligned}
& \int_{\mathcal{I}}\left(I_{t}^{+}(z)+I_{t}^{-}(z)\right) d z<\infty \text {, almost surely, and } \\
& I_{t}(z) \text { is decreasing in } z \text { for } \mathbb{P} \otimes d z \text {-almost every }(\omega, z) .
\end{aligned}
$$

Here $I_{t}^{+}(z)$ and $I_{t}^{-}(z)$ are defined by

$$
I_{t}^{+}:=\sum_{n>0, \kappa_{n}=1}^{\infty} 1_{\left\{\tau_{n}<t\right\}}, \quad I_{0}^{+}=0 \quad \text { and } \quad I_{t}^{-}:=\sum_{n>0, \kappa_{n}=0}^{\infty} 1_{\left\{\tau_{n}<t\right\}}, \quad I_{0}^{-}=0 .
$$

Definition 11 A singular control $\left(\xi^{+}, \xi^{-}\right)$is integrable if

$$
\mathbb{E}\left[\int_{0}^{\infty} e^{-\rho t}\left|\tilde{H}\left(Y_{t}\right) P_{t}\right| d t+\int_{[0, \infty)} e^{-\rho t}\left|K^{+}\right| d \xi_{t}^{+}+\int_{[0, \infty)} e^{-\rho t}\left|K^{-}\right| d \xi_{t}^{-}\right]<\infty
$$

\subsection{Solving $V(p, y)$}

Now, we solve $V(p, y)$ explicitly step by step.

Step 1: Singular control $\rightarrow$ switching control

Lemma 12 The value function in problem (11) is given by

$$
V(p, y)=\int_{0}^{y} v_{1}(p, z) d z+\int_{y}^{b} v_{0}(p, z) d z
$$

where $v_{0}$ and $v_{1}$ are solutions to the following optimal switching problems

$$
v_{k}(p, z):=\sup _{\alpha \in \mathcal{B} \text { limits }_{\kappa_{0}=k}} \mathbb{E}\left[\int_{0}^{\infty} e^{-\rho t}\left[\tilde{h}(z) P_{t}\right] I_{t} d t-\sum_{n=1}^{\infty} e^{-\rho \tau_{n}} K_{\kappa_{n}}\right],
$$

provided that the corresponding optimal switching controls are consistent and the resulting singular control is integrable. Here, $\alpha=\left(\tau_{n}, \kappa_{n}\right)_{n \geq 0}$ is an admissible two-state switching control, $\mathcal{B}$ is the subset of admissible switching controls $\alpha=$ $\left(\tau_{n}, \kappa_{n}\right)_{n \geq 0}$ such that $\left.\mathbb{E}\left[\sum_{n=1}^{\infty} e^{-\rho \tau_{n}}\right]<\infty\right\}$, with $K_{0}=K^{-}-\frac{C_{h}}{\rho}, K_{1}=K^{+}+\frac{C_{h}}{\rho}$, and $I_{t}$ the regime indicator function for any given $\alpha \in \mathcal{B}$.

The detailed proof of this lemma can be found in Guo and Tomecek (2008). It is worth pointing out that this lemma enables us to translate our original control problem 
to a two-state switching control problem between two regimes 0 and 1: for a given inventory level $z$, switching from state 0 to 1 corresponds to inventory increase and switching from state 1 to 0 corresponds to inventory decrease. The cost for inventory increase and decrease is given by $K^{+}+\frac{C_{h}}{\rho}$ and $-K^{-}+\frac{C_{h}}{\rho}$ respectively, and the benefit of being at state 1 is accumulated at rate $\tilde{h}(y)$. Furthermore, if there exists a consistent collection of switching controls so that the resulting singular control is integrable, then we have

$$
V(p, y)=\int_{y}^{b} v_{0}(p, z) d z+\int_{0}^{y} v_{1}(p, z) d z .
$$

where $v_{0}$ and $v_{1}$ are the corresponding value functions for switching controls. Moreover, explicit solution to $v_{0}$ and $v_{1}$ can be described analytically according to Ly Vath and Pham (2007). (We discuss this approach in more detail in Sect. 4.6.)

\section{Step 2: Solving switching controls and $v_{0}, v_{1}$}

Proposition $13 v_{0}$ and $v_{1}$ are the unique $C^{1}$ viscosity solutions with linear growth condition to the following system of variational inequalities:

$$
\begin{array}{r}
\min \left\{-\mathcal{L} v_{0}(p, z), v_{0}(p, z)-v_{1}(p, z)+K^{+}+\frac{C_{h}}{\rho}\right\}=0, \\
\min \left\{-\mathcal{L} v_{1}(p, z)-\tilde{h}(z) p, v_{1}(p, z)-v_{0}(p, z)+K^{-}-\frac{C_{h}}{\rho}\right\}=0,
\end{array}
$$

with boundary conditions $v_{0}\left(0^{+}, z\right)=0$ and $v_{1}\left(0^{+}, z\right)=\max \left\{-K^{-}+\frac{C_{h}}{\rho}, 0\right\}$. Here $\mathcal{L} u(p, z)=\sigma^{2} u_{p p}(p, z)+\mu u_{p}(p, z)-\rho u(p, z)$ where $u_{p}$ is the first derivative of $u$ with respect to $p$ and $u_{p p}$ is the second derivative of $u$ with respect to $p$.

This follows directly from Ly Vath and Pham (2007). To solve for $v_{0}, v_{1}$, we see by modifying the argument in Ly Vath and Pham (2007, Theorem 3.1) that for any given $z \in[0, b]$ and $k \in\{0,1\}$, an optimal switching control exists and can be described in terms of switching regions: there exist $0<F(z)<G(z)<\infty$ such that it is optimal to switch from regime 0 to regime 1 (to increase the inventory at level $z$ ) when $P_{t} \in[G(z), \infty)$, and to switch from regime 1 to regime 0 (decrease the inventory at level $z$ ) when $P_{t} \in[0, F(z)]$. Furthermore, based on Ly Vath and Pham (2007, Theorem 4.2), we see that for each $z \in[0, b]$, the switching regions are described in terms of $F(z)$ and $G(z)$, which take values in $(0, \infty]$ and can be explicitly derived as follows.

Case I: $K^{-}-\frac{C_{h}}{\rho} \geq 0$. First, for each $z \in[0, b]$ such that $\tilde{h}(z)=0$, it is never optimal to do anything, so we take $F(z)=\infty=G(z)$, and $v_{0}(p, z)=0=v_{1}(p, z)$.

Secondly, for $z$ such that $\tilde{h}(z)>0, G(z)<\infty$ and it is optimal to switch from regime 0 to regime 1 (to increase the inventory at level $z$ ) when $P_{t} \in[G(z), \infty)$. Since $K^{-}-\frac{C_{h}}{\rho} \geq 0$, it is never optimal to switch from regime 1 to regime 0 (i.e. $F(z)=\infty$ ). 
Furthermore, we have

$$
\begin{aligned}
& v_{0}(p, z)= \begin{cases}A(z) p^{n}, & p<G(z), \\
\eta \tilde{h}(z) p-\left(K^{+}+\frac{C_{h}}{\rho}\right), & p \geq G(z),\end{cases} \\
& v_{1}(p, z)=\eta \tilde{h}(z) p .
\end{aligned}
$$

Since $v_{0}$ is $C^{1}$ at $G(z)$, we get

$$
\left\{\begin{array}{l}
A(z) G(z)^{n}=\eta \tilde{h}(z) G(z)-\left(K^{+}+\frac{C_{h}}{\rho}\right) \\
n A(z) G(z)^{n-1}=\eta \tilde{h}(z) .
\end{array}\right.
$$

That is,

$$
\left\{\begin{array}{l}
G(z)=\frac{v}{\tilde{h}(z)} \\
A(z)=\frac{K^{+}+\frac{C_{h}}{\rho}}{(n-1)} G(z)^{-n}=\frac{K^{+}+\frac{C_{h}}{\rho}}{(n-1)} v^{-n} \tilde{h}(z)^{n},
\end{array}\right.
$$

where $v=\left(K^{+}+\frac{C_{h}}{\rho}\right) \sigma^{2} n(1-m)$.

Finally, when $\tilde{h}(z)<0$, it is optimal to switch from regime 1 to regime 0 (reduce inventory at level $z$ ) when $P_{t} \in[F(z), \infty)$. Since $K^{+}+\frac{C_{h}}{\rho}>0$, it is never optimal to switch from regime 0 to regime 1 (i.e. $G(z)=\infty$ ). The derivation of the value function proceeds analogously to the derivation for the case of $\tilde{h}(z)>0$.

Case II: $K^{-}-\frac{C_{h}}{\rho}<0$. First of all, for each $z \in[0, b]$ such that $\tilde{h}(z) \leq 0$, it is always optimal to reduce the inventory because $K^{-}-\frac{C_{h}}{\rho}<0$. That is, $F(z)=\infty=G(z)$. In this case, clearly $v_{0}(p, z)=0$ and $v_{1}(p, z)=-K^{-}+\frac{C_{h}}{\rho}$.

Next, for each $z \in[0, b]$ such that $\tilde{h}(z)>0$, it is optimal to switch from regime 0 to regime 1 (i.e. to increase in the inventory at level $z$ ) when $P_{t} \in[G(z), \infty)$, and to switch from regime 1 to regime 0 (i.e. to decrease in the inventory at level $z$ ) when $P_{t} \in(0, F(z)]$, where $0<F(z)<G(z)<\infty$.

Moreover, $v_{0}$ and $v_{1}$ are given by

$$
\begin{aligned}
& v_{0}(p, z)= \begin{cases}A(z) p^{n}, & p<G(z), \\
B(z) p^{m}+\eta x \tilde{h}(z)-\left(K^{+}+\frac{C_{h}}{\rho}\right), & p \geq G(z),\end{cases} \\
& v_{1}(p, z)= \begin{cases}A(z) p^{n}-\left(K^{-}-\frac{C_{h}}{\rho}\right), & p \leq F(z), \\
B(z) p^{m}+\eta p \tilde{h}(z), & p>F(z) .\end{cases}
\end{aligned}
$$


Smoothness of $V(p, z)$ at $p=G(z)$ and $p=F(z)$ leads to

$$
\left\{\begin{array}{l}
A(z) G(z)^{n}=B(z) G(z)^{m}+\eta G(z) \tilde{h}(z)-\left(K^{+}+\frac{C_{h}}{\rho}\right), \\
n A(z) G(z)^{n-1}=m B(z) G(z)^{m-1}+\eta \tilde{h}(z) \\
A(z) F(z)^{n}=B(z) F(z)^{m}+\eta F(z) \tilde{h}(z)+\left(K^{-}-\frac{C_{h}}{\rho}\right) \\
n A(z) F(z)^{n-1}=m B(z) F(z)^{m-1}+\eta \tilde{h}(z) .
\end{array}\right.
$$

Eliminating $A(z)$ and $B(z)$ from (28) yields

$$
\left\{\begin{array}{l}
\left(K^{+}+\frac{C_{h}}{\rho}\right) G(z)^{-m}+\left(K^{-}-\frac{C_{h}}{\rho}\right) F(z)^{-m}=\frac{-m}{(1-m) \rho} \tilde{h}(z)\left(G(z)^{1-m}-F(z)^{1-m}\right), \\
\left(K^{+}+\frac{C_{h}}{\rho}\right) G(z)^{-n}+\left(K^{-}-\frac{C_{h}}{\rho}\right) F(z)^{-n}=\frac{n}{(n-1) \rho} \tilde{h}(z)\left(G(z)^{1-n}-F(z)^{1-n}\right) .
\end{array}\right.
$$

Since the viscosity solutions to the variational inequalities are unique and $C^{1}$, for every $z$ there is a unique solution $F(z)<G(z)$ to (29). Let $\kappa(z)=F(z) \tilde{h}(z), v(z)=$ $G(z) \tilde{h}(z)$, then the following system of equations for $\kappa(z)$ and $v(z)$ is guaranteed to have a unique solution for each $z$ :

$$
\left\{\begin{array}{l}
\left(K^{+}+\frac{C_{h}}{\rho}\right) \nu(z)^{-m}+\left(K^{-}-\frac{C_{h}}{\rho}\right) \kappa(z)^{-m}=\frac{-m}{(1-m) \rho}\left(\nu(z)^{1-m}-\kappa(z)^{1-m}\right), \\
\left(K^{+}+\frac{C_{h}}{\rho}\right) \nu(z)^{-n}+\left(K^{-}-\frac{C_{h}}{\rho}\right) \kappa(z)^{-n}=\frac{n}{(n-1) \rho}\left(\nu(z)^{1-n}-\kappa(z)^{1-n}\right) .
\end{array}\right.
$$

Moreover, these equations depend on $z$ only through $v(z)$ and $\kappa(z)$, implying that there exist unique constants $\kappa, v$ such that $\kappa(z) \equiv \kappa$ and $v(z) \equiv v$ for all $z$. Hence $F(z)=\kappa \tilde{h}(z)^{-1}, \quad G(z)=v \tilde{h}(z)^{-1}$, with $\kappa<v$ being the unique solutions to

$$
\left\{\begin{array}{l}
\frac{1}{1-m}\left[v^{1-m}-\kappa^{1-m}\right]=-\frac{\rho}{m}\left[\left(K^{+}+\frac{C_{h}}{\rho}\right) v^{-m}+\left(K^{-}-\frac{C_{h}}{\rho}\right) \kappa^{-m}\right], \\
\frac{1}{n-1}\left[v^{1-n}-\kappa^{1-n}\right]=\frac{\rho}{n}\left[\left(K^{+}+\frac{C_{h}}{\rho}\right) v^{-n}+\left(K^{-}-\frac{C_{h}}{\rho}\right) \kappa^{-n}\right] .
\end{array}\right.
$$

Given $F(z)$ and $G(z), A(z)$ and $B(z)$ are solved from Eq. (28),

$$
\left\{\begin{array}{l}
B(z)=-\frac{G(z)^{-m}}{n-m}\left(\frac{G(z) \tilde{h}(z)}{\sigma^{2}(1-m)}-n\left(K^{+}+\frac{C_{h}}{\rho}\right)\right)=-\frac{F(z)^{-m}}{n-m}\left(\frac{F(z) \tilde{h}(z)}{\sigma^{2}(1-m)}+n\left(K^{-}-\frac{C_{h}}{\rho}\right)\right), \\
A(z)=\frac{G(z)^{-n}}{n-m}\left(\frac{G(z) \tilde{h}(z)}{\sigma^{2}(n-1)}+m\left(K^{+}+\frac{C_{h}}{\rho}\right)\right)=\frac{F(z)^{-n}}{n-m}\left(\frac{F(z) \tilde{h}(z)}{\sigma^{2}(n-1)}-m\left(K^{-}-\frac{C_{h}}{\rho}\right)\right) .
\end{array}\right.
$$

Step 3: Establishing the optimal control According to Lemma 12, it suffices to check the consistency of the switching control and the integrability of the corresponding singular control.

First, given the solution to the switching problems, clearly the optimal switching control for any level $z \in(0, b)$ is given by the following:

Case I: For $z \in(0, b)$ and $p>0$, let $F$ and $G$ be as given in for Case I. The switching control $\hat{\alpha}_{k}(p, z)=\left(\hat{\tau}_{n}(p, z), \hat{\kappa}_{n}(z)\right)_{n \geq 0}$, starting from $\hat{\tau}_{0}(p, z)=0$ and $\hat{\kappa}_{0}(z)=k$ is given by, for $n \geq 1$ 
- If $k=0, \hat{\tau}_{1}(p, z)=\inf \left\{t>0: P_{t} \in[G(z), \infty)\right\}$ and for $n \geq$ $2, \hat{\tau}_{n}(z)=\infty$

- If $k=1, \hat{\tau}_{1}(p, z)=\inf \left\{t>0: P_{t} \in[F(z), \infty)\right\}$ and for $n \geq$ 2, $\hat{\tau}_{n}(z)=\infty$.

Case II: For $z \in(0, b)$ and $p>0, F$ and $G$ as given for case II. The switching control $\hat{\alpha}_{k}(p, z)=\left(\hat{\tau}_{n}(p, z), \hat{\kappa}_{n}(z)\right)_{n \geq 0}$, starting from $\hat{\tau}_{0}(p, z)=0$ and $\hat{\kappa}_{0}(z)=k$ is given by, for $n \geq 1$

- If $\hat{\kappa}_{n-1}(z)=0, \hat{\tau}_{n}(p, z)=\inf \left\{t>\tau_{n-1}: X_{t}^{x} \in[G(z), \infty)\right\}, \hat{\kappa}_{n}(z)$ $=1$.

$-\quad$ If $\hat{\kappa}_{n-1}(z)=1, \hat{\tau}_{n}(p, z)=\inf \left\{t>\tau_{n-1}: X_{t}^{x} \in(0, F(z)]\right\}, \hat{\kappa}_{n}(z)=$ 0 ,

Now, define the collection of admissible switching controls $(\hat{\alpha}(p, z))_{z \in(0, b)}$ so that $\hat{\alpha}(p, z)=\hat{\alpha}_{0}(p, z)$ for $z>y$ and $\hat{\alpha}(p, z)=\hat{\alpha}_{1}(p, z)$ for $z \leq y$. Then,

Proposition 14 The collection of switching controls $(\hat{\alpha}(p, z))_{z \in(0, b)}$ is consistent.

Proof The consistency follows immediately from Definition 10 and the following monotonicity property of $F$ and $G: F$ is non-increasing and $G$ is non-decreasing in Case I, and $F$ is non-decreasing and $G$ is non-increasing in Case II.

Next, this consistent collection of optimal switching control corresponds to an admissible singular control $\left(\hat{\xi}^{+}, \hat{\xi}^{-}\right) \in \mathcal{A}_{y}$ in the following way according to Guo and Tomecek (2008).

Lemma 15 (From Switching Controls to Singular Controls) Given $y \in(0, b)$ and $a$ consistent collection of switching controls $(\hat{\alpha}(z))_{z \in \mathcal{I}}$, define two processes $\hat{\xi}^{+}$and $\hat{\xi}^{-}$ by setting $\hat{\xi}_{0}^{+}=0, \hat{\xi}_{0}^{-}=0$, and for $t>0: \hat{\xi}_{t}^{+}:=\int_{\mathcal{I}} I_{t}^{+}(z) d z, \hat{\xi}_{t}^{-}:=\int_{\mathcal{I}} I_{t}^{-}(z) d z$.

Then

1. The pair $\left(\hat{\xi}^{+}, \hat{\xi}^{-}\right) \in \mathcal{A}_{y}$ is an admissible singular control,

2. For all $t$, we almost surely have

$$
\hat{Y}_{t}=\operatorname{ess} \sup \left\{z \in \mathcal{I}: I_{t}(z)=1\right\}=\operatorname{essinf}\left\{z \in \mathcal{I}: I_{t}(z)=0\right\},
$$

where $\operatorname{ess} \sup \emptyset:=\inf \mathcal{I}$ and $\operatorname{ess} \inf \emptyset:=\sup \mathcal{I}$.

Moreover,

Proposition 16 The corresponding admissible singular control $\left(\hat{\xi}^{+}, \hat{\xi}^{-}\right) \in \mathcal{A}_{y}$ is integrable.

This is clear from Lemma 1 and Eq. (23) in Merhi and Zervos (2007).

Step 4. Solution Combining these results, we see that the ordering region is given by $\{(p, z): p \geq G(z)\}$ and the downsizing region by $\{(p, z): p \leq F(z)\}$. It is optimal to take no action in the continuation region, given by $\{(p, z): F(z)<p<G(z)\}$. If $(p, y)$ is in the ordering (or downsizing) region, then a jump is exerted at time zero to make $\hat{Y}_{0+}=G^{-1}(p)\left(\right.$ or $\left.\hat{Y}_{0+}=F^{-1}(p)\right)$. 
Finally, by Guo and Tomecek (2008, Theorem 3.10), we have

$$
V(p, y)=\int_{y}^{b} v_{0}(p, z) d z+\int_{0}^{y} v_{1}(p, z) d z,
$$

with

$$
\begin{aligned}
& v_{0}(p, z)= \begin{cases}A(z) p^{n}, & p<G(z), \\
B(z) p^{m}+\eta \tilde{h}(z) p-K^{+}-\frac{C_{h}}{\rho}, & p \geq G(z),\end{cases} \\
& v_{1}(p, z)= \begin{cases}A(z) p^{n}-K^{-}+\frac{C_{h}}{\rho}, & p \leq F(z), \\
B(z) p^{m}+\eta \tilde{h}(z) p, & p>F(z) .\end{cases}
\end{aligned}
$$

\subsection{Main result}

In summary, we see that the optimal value function is characterized below for two distinct cases. In the first, $K^{-}-\frac{C_{h}}{\rho} \geq 0$, implying that the proportional loss incurred upon selling inventory is greater than the gain from reduced future holding cost. In the second, $K^{-}-\frac{C_{h}}{\rho}<0$, implying that reducing holding cost dominates the transaction cost.

Theorem 17 [Optimal value function for $K^{-}-\frac{C_{h}}{\rho} \geq 0$ ]

$$
V(p, y)=\int_{0}^{y} v_{1}(p, z) d z+\int_{y}^{b} v_{0}(p, z) d z,
$$

where $v_{0}$ and $v_{1}$ are given by

1. For each $z \in[0, b]$ such that $\tilde{h}(z)=0: v_{0}(p, z)=v_{1}(p, z)=0$.

2. For each $z \in[0, b]$ such that $\tilde{h}(z)>0$ :

$$
\left\{\begin{array}{l}
v_{0}(p, z)= \begin{cases}A(z) p^{n}, & p<G(z), \\
\eta \tilde{h}(z) p-K^{+}-\frac{C_{h}}{\rho}, & p \geq G(z),\end{cases} \\
v_{1}(p, z)=\eta \tilde{h}(z) p,
\end{array}\right.
$$

where $G(z)=\frac{v}{\tilde{h}(z)}$, and $A(z)=\frac{K^{+}+\frac{C_{h}}{\rho}}{(n-1)} G^{-n}(z)$, with $v=\left(K^{+}+\frac{C_{h}}{\rho}\right) \sigma^{2} n(1-m)$.

3. For each $z \in[0, b]$ such that $\tilde{h}(z)<0$ :

$$
\left\{\begin{array}{l}
v_{0}(p, z)=0, \\
v_{1}(p, z)= \begin{cases}B(z) p^{n}+\eta \tilde{h}(z) p, & p<F(z), \\
-K^{-}+\frac{C_{h}}{\rho}, & p \geq F(z),\end{cases}
\end{array}\right.
$$


where $F(z)=-\frac{\kappa}{\tilde{h}(z)}$, and $B(z)=\frac{K^{-}-\frac{C_{h}}{\rho}}{(n-1)} \kappa^{-n} F^{-n}(z)$, with $\kappa=\left(K^{-}-\right.$ $\left.\frac{C_{h}}{\rho}\right) \sigma^{2} n(1-m)$.

Theorem 18 [Optimal value function for $K^{-}-\frac{C_{h}}{\rho}<0$ ]

$$
V(p, y)=\int_{0}^{y} v_{1}(p, z) d z+\int_{y}^{b} v_{0}(p, z) d z,
$$

where $v_{0}$ and $v_{1}$ are given by

1. For each $z \in[0, b]$ such that $\tilde{h}(z) \leq 0: v_{0}(p, z)=0, v_{1}(p, z)=-K^{-}+\frac{C_{h}}{\rho}$.

2. For each $z \in[0, b]$ such that $\tilde{h}(z)>0$ :

$$
\begin{aligned}
& v_{0}(p, z)= \begin{cases}A(z) p^{n}, & p<G(z), \\
B(z) p^{m}+\eta \tilde{h}(z) p-K^{+}-\frac{C_{h}}{\rho}, & p \geq G(z),\end{cases} \\
& v_{1}(p, z)= \begin{cases}A(z) p^{n}-K^{-}+\frac{C_{h}}{\rho}, & p \leq F(z), \\
B(z) p^{m}+\eta \tilde{h}(z) p, & p>F(z) .\end{cases}
\end{aligned}
$$

Here

$$
\begin{aligned}
& A(z)=\frac{\tilde{h}(z)^{n}}{(n-m) v^{n}}\left(\frac{v}{\sigma^{2}(n-1)}+m\left(K^{+}+\frac{C_{h}}{\rho}\right)\right) \\
& B(z)=\frac{-\tilde{h}(z)^{m}}{(n-m) v^{m}}\left(\frac{v}{\sigma^{2}(1-m)}-n\left(K^{+}+\frac{C_{h}}{\rho}\right)\right) .
\end{aligned}
$$

The functions $F$ and $G$ are non-decreasing with

$$
F(z)=\frac{\kappa}{\tilde{h}(z)} \quad \text { and } \quad G(z)=\frac{v}{\tilde{h}(z)},
$$

where $\kappa<v$ are the unique solutions to

$$
\begin{aligned}
& \frac{1}{1-m}\left[v^{1-m}-\kappa^{1-m}\right]=-\frac{\rho}{m}\left[\left(K^{+}+\frac{C_{h}}{\rho}\right) v^{-m}+\left(K^{-}-\frac{C_{h}}{\rho}\right) \kappa^{-m}\right], \\
& \frac{1}{n-1}\left[v^{1-n}-\kappa^{1-n}\right]=\frac{\rho}{n}\left[\left(K^{+}+\frac{C_{h}}{\rho}\right) v^{-n}+\left(K^{-}-\frac{C_{h}}{\rho}\right) \kappa^{-n}\right] .
\end{aligned}
$$

Theorem 19 [Optimal control for $K^{-}-\frac{C_{h}}{\rho} \geq 0$ ] For each $z \in[0, b]$, the optimal control is described in terms of $F(z)$ and $G(z)$ from Theorem 17 such that

- For $z$ such that $\tilde{h}(z)>0$, it is optimal to from regime 0 to regime 1 increase inventory past level $z$ when $P_{t} \in[G(z), \infty)$, and never decreases. 


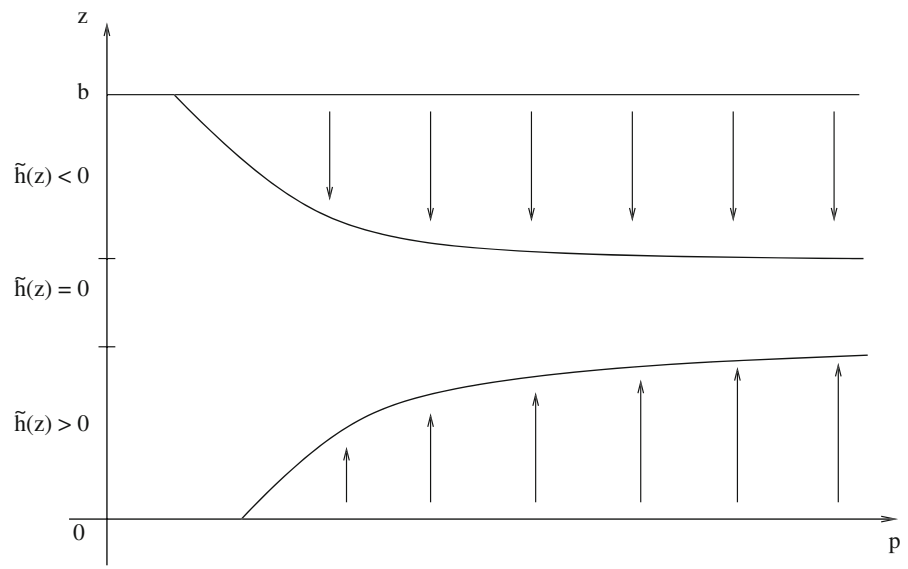

Fig. 1 Policy when $K^{-}-\frac{C_{h}}{\rho} \geq 0$, with $F(z)=-\frac{\kappa}{\tilde{h}(z)}$ and $G(z)=\frac{v}{\tilde{h}(z)}$

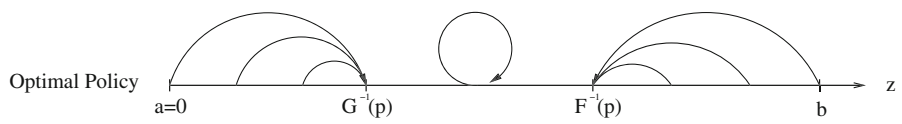

Fig. 2 Illustration of the two-threshold order policy for fixed price $p$ when $K^{-}-\frac{C_{h}}{\rho} \geq 0$

- When $\tilde{h}(z)<0$, it is optimal to decrease below inventory level $z$ when $P_{t} \in$ $[F(z), \infty)$, and it is never optimal to increase. When $\tilde{h}(z)=0$, it is optimal to do nothing (i.e. $F(z)=\infty=G(z)$ ).

Theorem 20 [Optimal control for $\left.K^{-}-\frac{C_{h}}{\rho}<0\right]$ For each $z \in[0, b]$, the optimal control is described in terms of $F(z)$ and $G(z)$ from Theorem 18 such that

- For $z$ such that $\tilde{h}(z)>0$, it is optimal to increase inventory past level $z$ when $P_{t} \in[G(z), \infty)$, and to decrease inventory below level $z$ when $P_{t} \in(0, F(z)]$.

- For $z$ such that $\tilde{h}(z) \leq 0$, it is always optimal to decrease inventory level.

The optimal policy is illustrated in Figs. 1 and 2 for the $K^{-} \geq \frac{C_{h}}{\rho}$ case, and Figs. 3 and 4 for the case of $K^{-}<\frac{C_{h}}{\rho}$. When $K^{-}<\frac{C_{h}}{\rho}$, implying a relatively high holding cost, for low prices inventory is decreased regardless of the value of $\tilde{h}(z)$; for high prices inventory is increased or decreased as necessary; for intermediate prices, no action is taken in general if inventory is low enough, but otherwise it is decreased (where Fig. 4 illustrates this last case). In contrast, when $K^{-} \geq \frac{C_{h}}{\rho}$, the relatively low holding cost introduces a different policy: above a certain threshold price, except around the $\tilde{h}(z)=0$ region, inventory is typically decreased for negative $h(z)$ values, and increased for positive $h(z)$ values, as illustrated in Fig. 2. In general, depending on the holding cost $C_{h}$ and the cost of selling $K^{-}$, reducing holding cost is a key driver, so conditions must be more favorable before inventory is increased, and it is more likely that inventory will be decreased. 


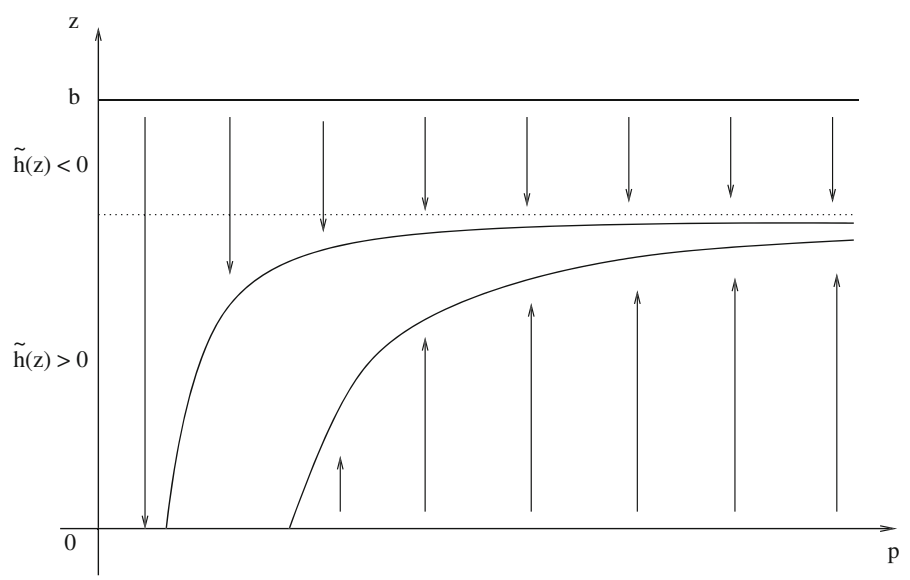

Fig. 3 Policy when $K^{-}-\frac{C_{h}}{\rho}<0$

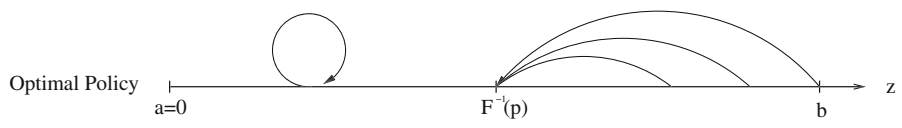

Fig. 4 Illustration of the one-threshold order policy for fixed (low) price $p$ and when $K^{-}-\frac{C_{h}}{\rho}<0$

Remark 21 We emphasize that these results are quite general, and indeed hold for any $\tilde{H}(\cdot)$ function that is concave. Thus, $F(z)$ and $G(z)$ are not necessarily continuous. Nevertheless, when $\tilde{H}$ is continuously differentiable, strictly increasing, and strictly concave, we will have the regularity condition for $F$ and $G$ and for the value function, as postulated in the current literature.

\subsection{Discussion}

As mentioned earlier, our solution approach is different from traditional approaches in which variational inequalities are solved directly.

To see this more closely, we first review the traditional dynamic programming approach and related variational inequalities. Clearly the optimization problem (11) for $V$ has the state space $\{p, z\}$. Given any price $p$ and the inventory level $z$ at time 0 , there are three options: do nothing, increase the inventory by purchasing on the spot market, or reduce the inventory by selling on the spot market.

If a quantity is purchased on the spot market, the inventory level jumps from $z$ to $z+\Delta z$, thus the value function is at least as good as choosing over all possible jumps of size $\Delta z$ with proportional cost $\left(K^{+}+\frac{C_{h}}{\rho}\right) \Delta z$. That is,

$$
V(p, z) \geq \sup _{\Delta z}\left(-\left(K^{+}+\frac{C_{h}}{\rho}\right) \Delta z+V(p, z+\Delta z)\right)
$$


which, by simple Ito's calculus, leads to $V_{y}(p, y) \leq K^{+}+\frac{C_{h}}{\rho}$, with $V_{y}(\cdot, \cdot)$ the derivative of the value function $V$ with respect to $y$. Similarly we see $V_{y}(p, y) \geq-K^{-}+\frac{C_{h}}{\rho}$ if choosing the option of reducing the inventory. Meanwhile, if no action is taken between time 0 and an infinitesimal amount of time $d t$, then expressing the value function at time 0 in terms of the value function at time $d t$ through dynamic programming and Ito's calculus (as in Constantinides and Richard 1978) yields $\sigma^{2} p^{2} V_{p p}(p, y)+$ $\mu p V_{p}(p, y)-r V(p, y)+\tilde{H}(y) \leq 0$, with $V_{p}(\cdot, \cdot)$ and $V_{p p}(\cdot, \cdot)$ the first and second derivatives respectively of the value function $V$ with respect to $p$. Combining these observations, we get the following (quasi)-Variational Inequalities

$$
\begin{array}{r}
\max \left\{\sigma^{2} p^{2} V_{p p}(p, y)+\mu p V_{p}(p, y)-r V(p, y)+\tilde{H}(y) p,\right. \\
\left.V_{y}(p, y)-K^{+}-\frac{C_{h}}{\rho},-V_{y}(p, y)-K^{-}+\frac{C_{h}}{\rho}\right\}=0 .
\end{array}
$$

Moreover, the optimal policy (if it exists) can be characterized by explicitly finding the action and continuation regions where

$$
\left\{\begin{array}{l}
(\text { Inventory increase region })=\left\{(p, y): V_{y}(p, y)=-K^{-}+\frac{C_{h}}{\rho}\right\}, \\
\text { (Inventory decresse region) }=\left\{(p, y): V_{y}(p, y)=K^{+}+\frac{C_{h}}{\rho}\right\}, \\
\text { (No action region) }=\quad\left\{(p, y): V_{y}(p, y)>-K^{-}+\frac{C_{h}}{\rho},\right. \\
\\
V_{y^{+}}(p, y)<K^{+}+\frac{C_{h}}{\rho}, \\
\sigma^{2} p^{2} V_{p p}(p, y)+\mu p V_{p}(p, y)-r V(p, y) \\
+\tilde{H}(y) p=0\} .
\end{array}\right.
$$

A typical explicitly solvable optimal policy is a two-threshold bang-bang type policy or some degenerate form. (For more detailed derivation and background, interested readers are referred to e.g., Constantinides and Richard 1978 or Harrison and Taksar 1983.)

Taking this analysis one step further, one would expect, due to the stochastic nature of the $P_{t}$, a state-dependent threshold policy, where inventory is lowered if it is above the upper threshold, and increased if it is below the lower threshold (see, e.g, Constantinides and Richard 1978). That is, we would expect a downsizing region for inventory: $\{(p, z): p \geq G(z)\}$, an ordering region: $\{(p, z): p \leq F(z)\}$, and a (continuation) no-action region: $\{(p, z): F(z)<p<G(z)\}$.

However, there is a serious issue in this straightforward extension. In order to derive a complete characterization of the optimal policy and the value function, one in general would assume a priori enough smoothness for the value function and the boundary to solve the QVI. Unfortunately, the regularity conditions for this two-dimensional control problem do not hold in general. (See counter-examples in Guo and Tomecek 2008). Indeed, the value function may not be $C^{1}$ in $p$ (although it is $C^{1}$ in $y$ ) and $F, G$ may not be continuous. This possible irregularity especially in $F$ and $G$ makes explicit solution or "guessing" of $F$ and $G$ particularly difficult. 
This is where we depart from the traditional approach: instead of solving variational inequalities directly, we translate the singular control problem (11) into a switching control problem, following Guo and Tomecek (2008). The key idea is that by fixing each level $z_{0}$, we effectively will be solving for a one-dimensional two-state switching control problem, where switching from 0 to 1 corresponds to inventory increases and switching from 1 to 0 represents inventory reduction. In order for this approach to work for all $z$, meaning we can break down the two-dimensional control problem by slicing it into pieces of one-dimensional problem, we need to make sure the resulting control policies at different levels of $z$ are "consistent". Intuitively, this consistency requires that for a given price $p$ at level $z_{0}$, if it is optimal to reduce the inventory level, then it is also optimal to reduce the inventory level given the same $p$ and a higher level $z\left(>z_{0}\right)$. This is the essence of Lemma 12 and our solution approach.

\section{Computational experiments and observations}

We have characterized the optimal solution for the problem faced by a firm facing random demand at the end of a single period of random length when that firm has the opportunity to trade on the spot market while waiting for demand. Of course, the opportunity to actively utilize the spot market to guard against cost and price risks must be balanced against the increasing complexities of actively trading inventory prior to experiencing demand. Below, we describe computational experiments that explore this tradeoff.

\subsection{Modified newsvendor}

To that end, we compare the expected results from the optimal policy as described in this paper to those based on the use of a modified newsvendor solution to this model. We elected to use this modified newsvendor approach as a reasonable proxy for how a manager who is not interested in repeatedly buying and selling on the spot market might manage the system.

In this modified newsvendor model, the manager purchases the inventory at the beginning of the investment, using a version of the well-known newsvendor solution adapted for the specifics of this setting. In particular, the newsvendor decision is based on the expected time until demand arrival, $E[\tau]$, the expected discounted sales price, $\alpha E\left[e^{-r \tau} p_{\tau}\right]$, and the expected discounted penalty cost and salvage value, $\alpha_{u} E\left[e^{-r \tau} p_{\tau}\right]$ and $\alpha_{o} E\left[e^{-r \tau} p_{\tau}\right]$. The modified newsvendor inventory level, $y^{*}$, is given as follows:

$$
y^{*}=\min \left\{b, \max \left\{0, F_{D}^{-1}\left(\frac{\left(\alpha+\alpha_{u}\right) E\left[e^{-r \tau} p_{\tau}\right]-\left(p_{0}+K^{+}+C_{h} E[\tau]\right)}{\left(\alpha-\alpha_{0}+\alpha_{u}\right) E\left[e^{-r \tau} p_{\tau}\right]}\right)\right\}\right\},
$$

where 0 and $b$ are the lower and upper bounds on the inventory level, $p_{0}+K^{+}+C_{h} E[\tau]$ is the expected cost per unit of acquiring (and holding) inventory, and $F_{D}$ is the cumulative distribution of the random demand, $D$. 
Recall $\tau$ is an exponential random variable with parameter $\lambda$, and $p_{t}$ is a geometric Brownian motion with drift $\mu$ and volatility $\sigma$. Thus, $E[\tau]=\frac{1}{\lambda}$ and $E\left[e^{-r \tau} p_{\tau}\right]=$ $\frac{\lambda p_{0}}{\lambda+r-\mu}$. Substituting, we get

$$
y^{*}=\min \left\{b, \max \left\{0, F_{D}^{-1}\left(\frac{\left(\alpha+\alpha_{u}\right) \lambda P_{0}-\left(p_{0}+K^{+}+C_{h} / \lambda\right)(\lambda+r-\mu)}{\left(\alpha-\alpha_{0}+\alpha_{u}\right) \lambda P_{0}}\right)\right\}\right\} .
$$

Finally, the expected value of implementing this modified newsvendor policy is

$$
\begin{aligned}
& E\left[\alpha e^{-r \tau} P_{\tau} \min \left(y^{*}, D\right)-\left(p_{0}+K^{+}+C_{h} \tau\right) y^{*}\right. \\
& \left.\quad+\alpha_{o} e^{-r \tau} P_{\tau} \max \left(y^{*}-D, 0\right)-\alpha_{u} e^{-r \tau} P_{\tau} \max \left(D-y^{*}, 0\right)\right]
\end{aligned}
$$

\subsection{Scenarios}

Recall that we have two different cases when characterizing the optimal value function: $K^{-} \geq C_{h} / \rho$, where the proportional loss incurred on selling inventory is greater than the gain from reduced future holding cost, and $K^{-}<C_{h} / \rho$, where reducing holding cost dominates transaction costs. Based on solution structures in Sect. 4.5, we consider three scenarios in these experiments:

Scenario $1 K^{-} \geq C_{h} / \rho$, and $y_{0}=0\left(h\left(y_{0}\right)>0\right)$, so that in the optimal solution the manager may buy more inventory before time $\tau$.

Scenario $2 K^{-} \geq C_{h} / \rho$, and $y_{0}=b\left(h\left(y_{0}\right)<0\right)$, so that in the optimal solution the manager may sell off excessive inventory before time $\tau$

Scenario $3 K^{-}<C_{h} / \rho$ (and $y_{0}=0$ ), so that in the optimal solution may buy more inventory or sell off excessive inventory before time $\tau$.

\subsection{Parameters}

For simplicity, we set the discount rate $r=0$, the demand arrival rate $\lambda=1$, the salvage multiplier $\alpha_{0}=0$, and the penalty cost multiplier $\alpha_{u}=0$. To create the three scenarios described above, we set the mark-up multiplier $\alpha=1.3$, the holding cost $C_{h}=1$, the transaction cost for buying $K^{+}=1$, for Scenarios 1 and 2 the transaction cost of selling $K^{-}=1.5$, and for Scenario 3 the transaction cost of selling $K^{-}=0.5$. We set the inventory upper bound $b=200$. To model the random demand, $D$, we assume $\log (D)$ follows a normal distribution with parameters $\mu_{d}=5$, and $\sigma_{d}=0.7$. Hence, the mean and the variance of $D$ are:

$$
\begin{gathered}
E[D]=e^{\mu_{d}+\frac{\sigma_{d}^{2}}{2}}=189.61, \\
\operatorname{var}[D]=\left(e^{\sigma_{d}^{2}}-1\right) e^{2 \mu_{d}+\sigma_{d}^{2}}=22734.40 .
\end{gathered}
$$




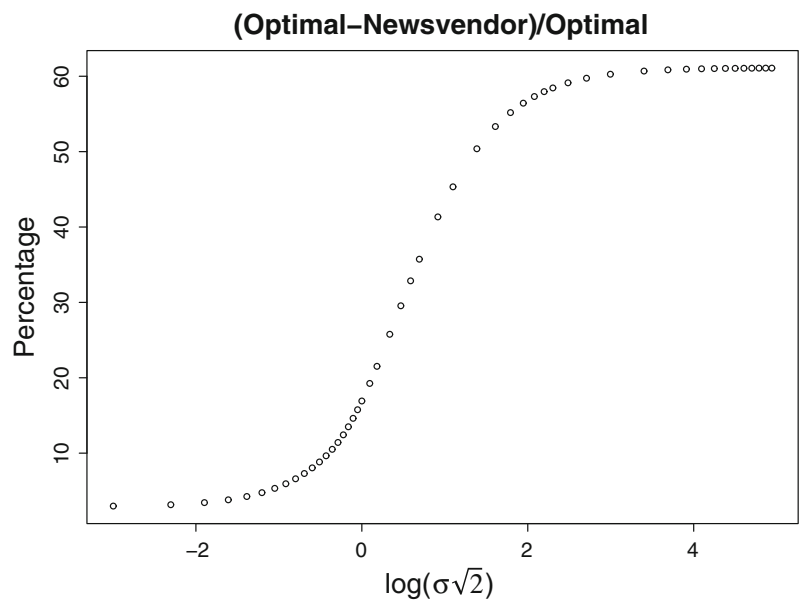

Fig. 5 Scenario 1, percentage difference versus change in price volatility

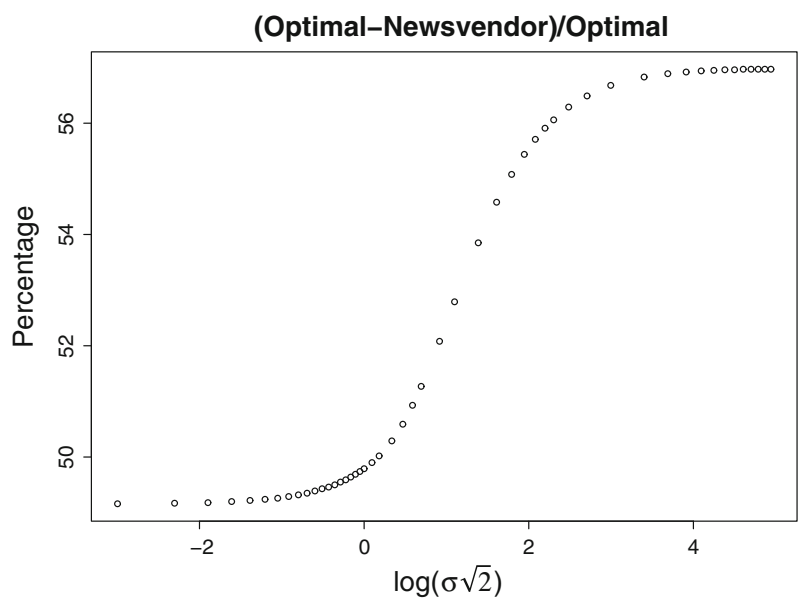

Fig. 6 Scenario 2, percentage difference versus change in price volatility

For the price process $p_{t}$, we set the initial price $p_{0}=5$, and the drift rate $\mu=0.3$. We vary the volatility $\sigma$ from $0.05 / \sqrt{2}$ to $140 / \sqrt{2}$ to assess the impact of volatility.

\subsection{Results and observations}

We use Theorems 4, 17 and 18 and the parameters listed above to calculate the optimal expected value for Scenarios 1, 2, and 3, and in Figs. 5, 6, and 7, we graph the relative gain from using the optimal policy rather than the modified newsvendor policy (that is, the difference between the optimal expected value and the newsvendor value divided by the optimal expected value) for different price volatilities. 


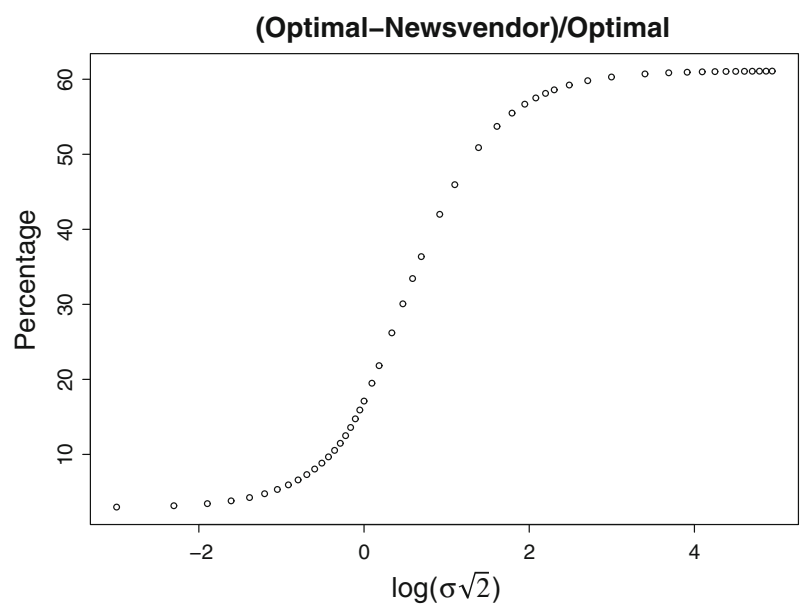

Fig. 7 Scenario 3, percentage difference versus change in price volatility

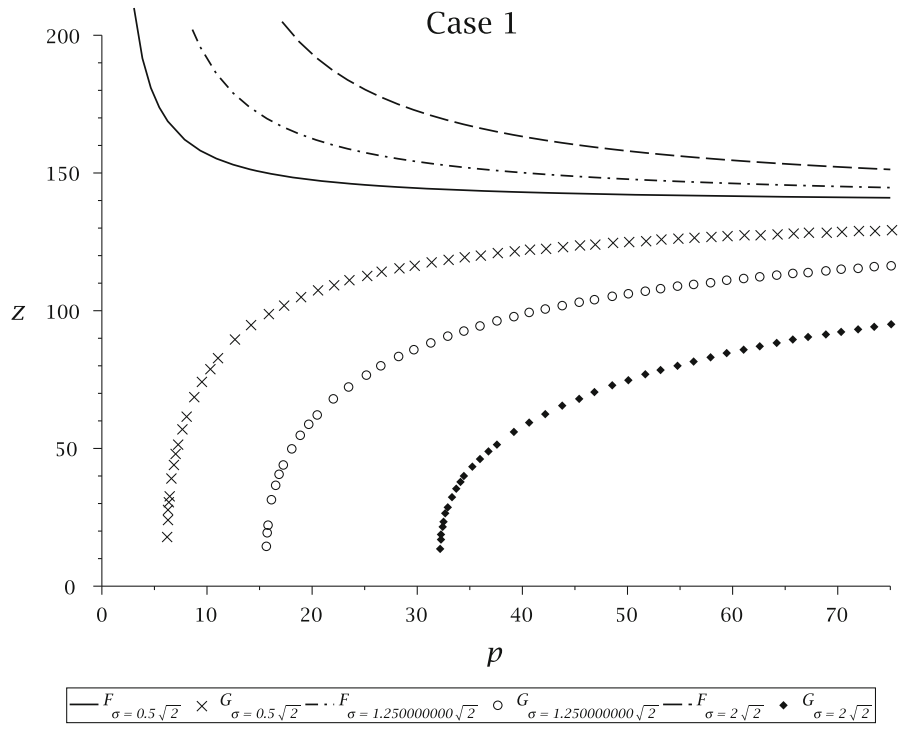

Fig. 8 Figure 1 redrawn for different price volatilities

For all three scenarios, the advantage of the optimal policy over the modified newsvendor policy increases rapidly then slowly saturates at around 55-60\% as $\sigma$ increases. To better understand the policy leading to these results, we redraw Figs. 1 and 3 for different price volatilities.

In Fig. 8, we observe that both $F^{-1}$ and $G^{-1}$ shift to the right as $\sigma$ increases. This suggests that as price volatility increases, the spot market price at which the manager should take action increases, enabling the manager to take advantage of this increased volatility. In addition, as volatility increases, $F^{-1}$ shifts upwards and $G^{-1}$ shifts downwards so that the "no action" region increases in size, suggesting more conservative 


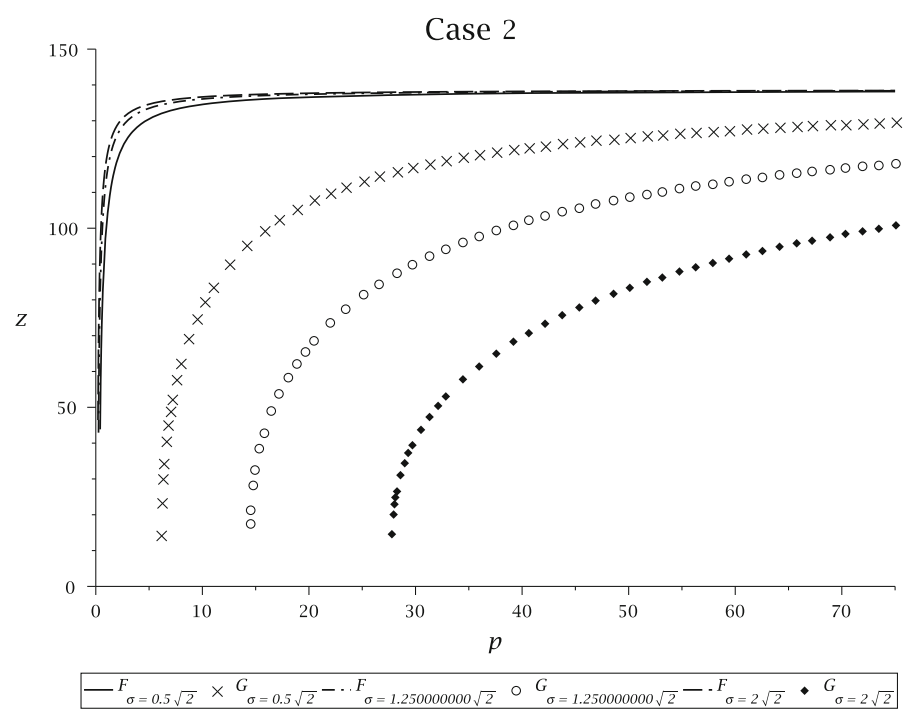

Fig. 9 Figure 3 redrawn for different price volatilities

behavior on the part of the manager. Similarly, in Fig. $9, G^{-1}$ shifts in a similar way as the volatility increases, again suggesting more "conservative" purchasing as volatility increases (that is, smaller amounts bought for a given price). However, $F^{-1}$ shifts up and to the left as volatility increases, implying that as volatility increases, the manager in this case should wait until the price process drops further down and make fewer adjustments as volatility increases.

\section{Future research}

We have completely characterized the optimal policy for a firm facing random demand after a random period of time of being able to buy and sell on the spot market. In computational tests, we observed that this policy performs significantly better than a version of the traditional newsboy policy (utilized as a proxy for a reasonable inventory management policy for firms not interested in trading on the spot market), most notably when price volatility is relatively high.

Although the results presented in this paper provide insight into the value to a firm of effectively utilizing the spot market, and contribute to the state of the art in continuous time inventory control, there are significant extensions possible to this work from both technical and modeling perspectives.

For example, the addition of a fixed inventory ordering cost changes the singular control to a more difficult impulse control problem. It will be interesting to see if the analogous state dependent version of $(d, D, U, u)$ policy still holds for this two-dimensional problem, and whether the regularity property holds as well. Additionally, the price process could be modeled by stochastic processes other than a Brownian motion. For instance, it would be interesting to explore whether or not the two-threshold policy 
holds for the case of a mean-reverting process. More sophisticated constraints on the inventory, such as a requirement that the inventory either be 0 or above some minimum level, are in theory not more difficult by our solution approach, but it would be interesting to complete this analysis. Finally, multi-period models, with multiple demand opportunities and inventory carried between periods will be significantly more difficult to analyze, but may yield interesting insights on effective inventory management in the presence of a spot market.

Open Access This article is distributed under the terms of the Creative Commons Attribution Noncommercial License which permits any noncommercial use, distribution, and reproduction in any medium, provided the original author(s) and source are credited.

\section{References}

Archibald A (1981) Continuous review (s,S)policies with lost sales. Manag Sci 27:1171-1177

Bather JA (1966) A continous time inventory model. J Appl Prob 3:538-549

Berling P (2008) The capital cost of holding inventory with stochastically mean-reverting purchase price. Eur J Oper Res 186:620-636

Billington C (2002) Hp cuts risk with portfolio approach. Purchasing Magazine Online

Chaouc B (2006) Inventory control and periodic price discounting campaigns. Naval Res Log 54:94-108

Chen F, Song J-S (2001) Optimal polices for multiechelon inventory problems with markov-modulated demand. Oper Res 49(2):226-234

Cheng F, Sethi S (1999) Optimality of state-dependent $(\mathrm{s}, \mathrm{S})$ policies in inventory models with markovmodulated demand and lost sales. Prod Oper Manag 8(2):183-192

Constantinides G, Richard S (1978) Existence of optimal simple policies for discounted-cost inventory and cash management in continuous time. Oper Res 26(4):620-636

Gavirneni S, Morton T (1999) Inventory control under speculation: myopic heuristics and exact procedures. Eur J Oper Res 117:211-221

Goh M, Sharafali M (2002) Price-dependent inventory models with discount offers at random times. Prod Oper Manag 11(2):139-156

Golabi K (1985) Optimal inventory policies when ordering prices are random. Oper Res 33(3):575-588

Guo X, Tomecek P (2008) Connections between singular control and optimal switching. SIAM J Control Optim 47(1):421-443

Haksoz C, Seshadri S (2007) Supply chain operations in the presence of spot market: a review with discussion. J Oper Res Soc (advance online publication 11)

Harrison JM, Selke TM, Taylor AJ (1983) Impulse control of Brownian motion. Math Oper Res 8(3):454466

Harrison JM, Taksar M (1983) Instantaneous control of brownian motion. Math Oper Res 8(3):439-453

Kalymon BA (1971) Stochastic prices in a single-item inventory purchasing model. Oper Res 19(6):14341458

Li C-L, Kouvelis P (1999) Flexible and risk-sharing contracts under price uncertainty. Manag Sci 45(10):1378-1398

Ly Vath V, Pham H (2007) Explicit solution to an optimal switching problem in the two-regime case. SIAM J Control Optim 46(2):395-426

Merhi A, Zervos M (2007) A model for reversible investment capacity expansion. SIAM J Control Optim 46:839-876

Moinzadeh K (1997) Replenishment and stocking policies for inventory systems with random deal offerings. Manag Sci 43(3):334-342

Ormeci A, Dai JG, Vate JV (2006) Impulse control of brownian motion: the constrained average cost case.

Protter PE (2004) Stochastic integration and differential equations. Springer, Berlin

Schainker RB (2004) Executive overview: energy storage options for a sustainable energy future. In: Proceedings of the Power Engineering Society General Meeting, vol 2, pp 2309-2314

Sethi SP, Cheng F (1997) Optimality of (s,s) policies in inventory models with markovian demand. Oper Res 45(6):931-939 
Simchi-Levi D, Kaminsky P, Simchi-Levi E (2008) Designing and managing the supply chain. McGrawHill, Boston

Sulem A (1986) A solvable one-diemensional model of a diffusion inventory system. Math Oper Res 11(1):125-133

Taksar MI (1985) Average optimal singular sontrol and a related stopping problem. Math Oper Res 10:63

Wang Y (2001) The optimality of myopic stocking policies for systems with decreasing prices. Eur J Oper Res 133:53-159 